\title{
Applicability of the planar Hall effect
}

\author{
Jan H. Fluitman \\ Twente University of Technology, Department of Electric Engineering, P.O.B. 217, 7500 AE, Enschede, \\ The Netherlands \\ The planar Hall effect in ferromagnetic films has been investigated. Particularly the response to \\ inhomogeneous fields has been studied in view of the method of detection of the Hall voltage ("one-sided" or \\ traditional). It is concluded that "one-sided" detection may be of advantage. The Hall response around the \\ theoretical singularity of the transverse susceptibility has been studied. It is found that a very sensitive device \\ can be designed, based on this effect.
}

PACS numbers: 06.70.Dn, 72.15.Gd, 73.60.Dt

INTRODUCTION

The magnetoresistance of permalloy strips is utilized in magnetic bubble memories (as bubble detection mechanism) and recently in a magnetic recording read head. The (planar) Hall effect is directly related to the magnetoresistance and consequently is also present in such devices. It has not had much attention recently and found no application as far as we know. Nevertheless the planar Hall resistivity has the adventage of being linear in first order, which is of importance particularly for a recording read head or a related application (e.g. position detector). The reason that the essentially quadratic magnetoresistance has been chosen for such applications may be twofold. At first the magnetoresistive voltage relates to the Hall voltage as $1 / \mathrm{w}$, the length/width ratio of the strip, and it is much easier to have a relative long strip in the region where the fields are than it is for a relative wide one. Secondly, a magnetoresistive transducer requires only two contact leads, while a Hall transducer in its traditional form with two voltage leads at opposite sides of the strip may be particularly cumbersome in cases that the transducer must have a position at the edge of a substratum. However, with respect to the latter a layout has been designed (hereafter denoted as "onesided", see figure 1), which may be helpful to solve that problem (1). With respect to the first reason we note that trackwidthes in magnetic recording become smaller and smalier, which means that the upper limit of 1 (length) decreases and this, together with the possibility to use relative wide transducers in shielded heads, can also bring the planar Hall effect into the picture. In the next sections we treat the theory and the necessary computational procedures. Then we give results of computations concerning the transducer response to exponential field distributions. Next we pay attention to the singular behaviour around $\mathrm{H}=\mathrm{H}_{\mathrm{k}}$, where the slope of the Hall curve approaches infinity and finally we give results of experiments, performed to test the main lines of the predicted behaviour.

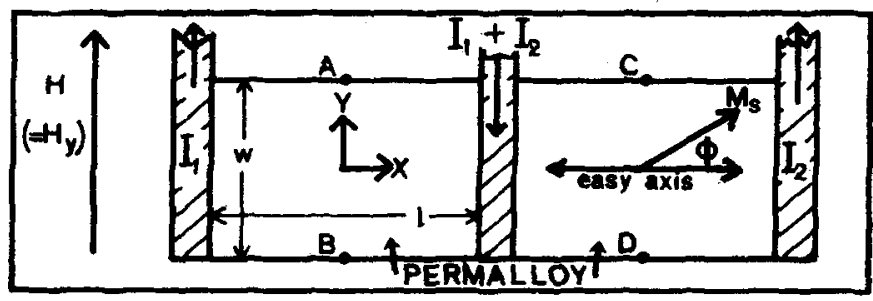

FIG. 2. Layout of a planar Hall transducer suitable for "one-sided" detection of the Hall voltage. The currents $I_{1}, I_{2}$ are choosen so that $V_{A C}=0$ (or $V_{D B}=0$ ) at $\mathrm{H}=0$.
THEORY

Expressions for the planar galvanomagnetic effects in conductive ferromagnetic films are given in the literature (2):

$$
\begin{aligned}
& \rho_{M}=\rho_{\|}-\left(\rho_{\|}-\rho_{\perp}\right) \sin ^{2} \phi \\
& \rho_{H}=\left(\rho_{y}-\rho_{\perp}\right) \sin \phi \cos \phi
\end{aligned}
$$

with $\phi$ the angle of rotation of the magnetization vector with respect to the axis of uniaxial anisotropy which we take along the strip axis. $\rho_{\| \prime}$ and $\rho_{\perp}$ are the resistivities parallel and perpendicular to the magnetization vector respectively. $\rho_{\mathrm{H}}$ is the Hall resistivity and $\rho_{M}$ the magnetoresistivity. Adopting the single domain model, with $\sin \phi=\mathrm{H} / \mathrm{H}$ for $|\mathrm{H}| \leq \mathrm{H}_{\mathrm{k}}$ and $\sin \phi= \pm 1$ for $|\mathrm{H}|>\mathrm{H}_{\mathrm{k}}\left(\mathrm{H}_{\mathrm{k}} \frac{\mathrm{k}}{1 \mathrm{~s}}\right.$ the

$$
\begin{aligned}
& \rho_{M}=\begin{array}{ll}
\rho_{\| \prime}-\left(\rho_{\|}-\rho_{\perp}\right)\left(H / H_{k}\right)^{2} & |H| \leq H_{k} \\
\rho_{\perp} & |H|>H_{k}
\end{array} \\
& \rho_{H}=\begin{array}{ll}
\left(\rho_{J}-\rho_{\perp}\right)\left(\mathrm{H} / \mathrm{H}_{\mathrm{k}}\right) \sqrt{1-\left(\mathrm{H} / \mathrm{H}_{\mathrm{k}}\right)^{2}} & |\mathrm{H}| \leq \mathrm{H}_{\mathrm{k}} \\
0 & |\mathrm{H}|>\mathrm{H}_{\mathrm{k}}
\end{array}
\end{aligned}
$$

These equations are valid for the case that the applied field $\mathrm{H}$, oriented perpendicular to the strip axis, but in the plane of the strip (fig.1), penetrates the strip. For the case that demagnetizing effects can not be ignored however, simple expressions only exist for strips with an elliptic cross-section. Then one simply can replace $H_{k}$ by the effective anisotropy field $\mathrm{H}_{\mathrm{k}}+\mathrm{tM} / \mathrm{w}$, where $\mathrm{t}$ (thickness) and $\mathrm{w}$ (width) are considered as the principal axes of the ellips. $M$ is the saturation magnetization. For the more realistic rectangular cross-section analytic expressions cannot be obtained.In that case one has to solve the equations:

$$
\begin{array}{cc}
H_{I}(y)=H(y)+\frac{M}{\pi^{S}}{ }_{-w / 2}^{w / 2} \frac{d \sin \phi\left(y_{0}\right)}{d y_{0}} \operatorname{arctg} \frac{t}{2\left(y_{0}-y\right)} d y_{0} \\
\sin \phi(y)=I I y) / H_{k} & \left|H_{I}(y)\right| \leq H_{k} \\
\pm 1 & \left|H_{I}(y)\right|>H_{k}
\end{array}
$$

where $H_{T}(y)$ stands for the field inside the strip. More details about the equations and their solution can be found in the literature (3). It is seen from Eq.5 that the externally applied field may depend on $y$ and that is what we need in studying responses to exponential fields. Eqs. (5) and (6) can be solved numerically and once $\phi(y)$ is known it is possible to compute any Hall potential. A local Hall potential is defined as the potential change due to the applied field, assuming fixed potentials at the current leads. 
Following the lines described by Hlasnik and Kokavec (4), we find:

$V_{\mathrm{H}}(\vec{r})=\frac{\left(\rho_{\mathrm{u}}-\rho_{\perp}\right) I}{2 \pi w t}-\mathrm{w} / 2 \sin \phi\left(\mathrm{y}_{0}\right) \cos \phi\left(y_{0}\right) \mathrm{G}\left(\mathrm{y}_{0}, \mathrm{x}, \mathrm{y}, \mathrm{l}, \mathrm{w}\right) \mathrm{dy} \mathrm{y}_{0}$

with I the current through the strip and $G$ a characteristic function which includes a- (second) form effect, due to the short circuiting of the Hall voltage under the current leads. This effect is wellknown and described for the normal Hall effect (5). Values for the G-function can also be found in the literature (1). For more clarity we give an overview of the computational procedure in fig.?.

\section{RESPONSE TTO EXPONENTIAL FIELDS}

We have choosen the exponentially decaying field $\mathrm{H}=\mathrm{H}_{\mathrm{O}} \exp (-\mathrm{y} / \mathrm{cw})$ as a test field to analyse the effect of "hne-sided" detection. We have normalized y to the width of the transducer and introduced $c$ as a measure of homogeneity. For $c=\infty$ we have the limiting case of homogeneous fields. There is no difference then between "one-sided" detection and the conventional method. The Hall voltages for this case are collected in fig. 3 for several values of the formfactors. In exponential fields the response depends on the method of detection. In fig. 4 it is seen that for small values of $\mathrm{H}_{0}$ the largest response is obtained with "one-sided" detection at that side, where the fields are largest. But on increase of $\mathrm{H}_{0}$ the transducer will saturate at that side first, which means that the signal from that side will also decrease as first. This effect is particularly pronounced when there is no

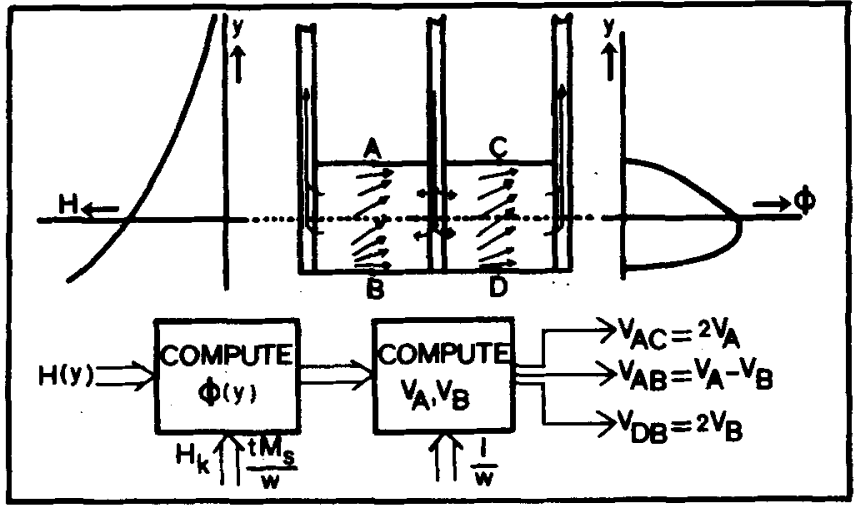

FIG.2.Scheme of the computational procedure.In fact onl the Hall potentials at points $A$ and $B$ are computed, from which the relevant voltage differences can be derived.

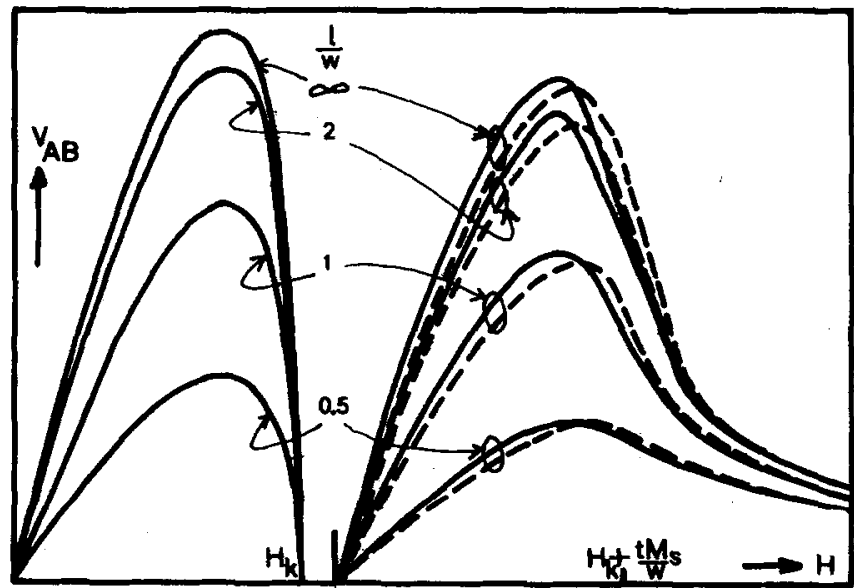

FIG.3.Hall voltages in homogeneous fields.At left for $\mathrm{tM}_{\mathrm{s}} / \mathrm{w}=0$ (no demagnetization), at right with $\mathrm{H}_{\mathrm{k}}=\mathrm{tM} / \mathrm{w}$ (drawn curves) and $\mathrm{H}_{\mathrm{k}}<\mathrm{t} \mathrm{M}$. $/ \mathrm{w}$ (dashed curves). Values for the formfactor $1 / \mathrm{w}$ are $\infty, \mathfrak{\xi}, 1$ and 0.5 (from up to down). demagnetization. Demagnetizing effects have the tendency to spread the fields inside the strip and suppress the differences between the several Hall voltages.

The most relevant parameter in our opinion is the Hall sensitivity at $\mathrm{H}=0$. In $\mathrm{fig} .5$ we have plotted this parameter, where we have normalized the amplitude $\mathrm{H}_{0}$ on $\mathrm{H}_{\mathrm{k}}+\mathrm{tM}_{\mathrm{s}} / \mathrm{w}$. The two points at the right side of the figure refer to the case of homogeneous fields. Again it can be seen that the differences are largest when the demagnetizing effects are smallest. Figures (4) and (5) are computed for $1 / w=\infty$. For short transducers with $1 / w=2$ all values are somewhat lower $(90-95 \%)$.

In our opinion the figures show that "one-sided" detection can be applied, without a disastrous loss in sensitivity to be paid for.

\section{SINGULARITY AT $\mathrm{H}=\mathrm{H}_{\mathrm{k}}$}

In strips with $\mathrm{H}_{\mathrm{k}}>\mathrm{tM}_{\mathrm{s}} / \mathrm{w}$ and for the case of homogeneous fields the $\mathrm{H}_{\mathrm{k}} \mathrm{ll}$ voltage falls sharply at $\mathrm{H}=\mathrm{H}_{\mathrm{k}}$. This phenomenon can be explained with the help of the geometrical properties of the Stoner-Wohlfarth astroid (6). In fig. 6 we have $\sin \phi=\left(\mathrm{H}_{\mathrm{k}}-\Delta \mathrm{H}_{\mathrm{y}}\right) / \mathrm{H}_{\mathrm{k}}$ and $\operatorname{ctg} \phi=\Delta \mathrm{H}_{\mathrm{x}} / \Delta \mathrm{H}_{\mathrm{y}}$ and from these it follows:

$$
\begin{aligned}
\Delta \mathrm{H}_{\mathrm{y}} & =\mathrm{H}_{\mathrm{k}}(1-\sin \phi) \\
\Delta \mathrm{H}_{\mathrm{x}} & =\mathrm{H}_{\mathrm{k}}(1-\sin \phi) \operatorname{ctg} \phi
\end{aligned}
$$

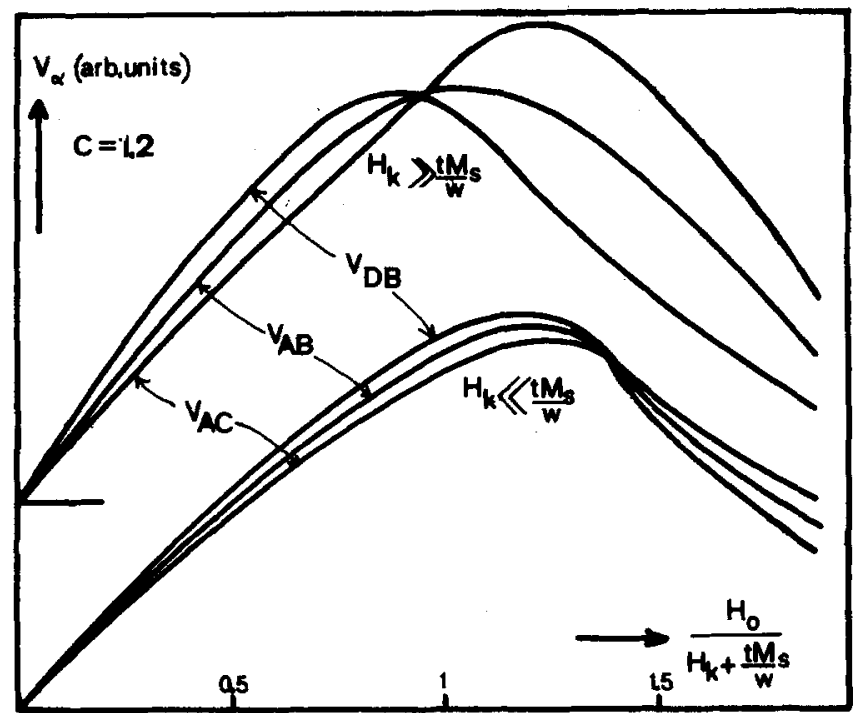

IG. 4. Characteristic response curves of the transducer placed in exponential fields. Typical are the "cross-over" points as the transducer approaches saturation.

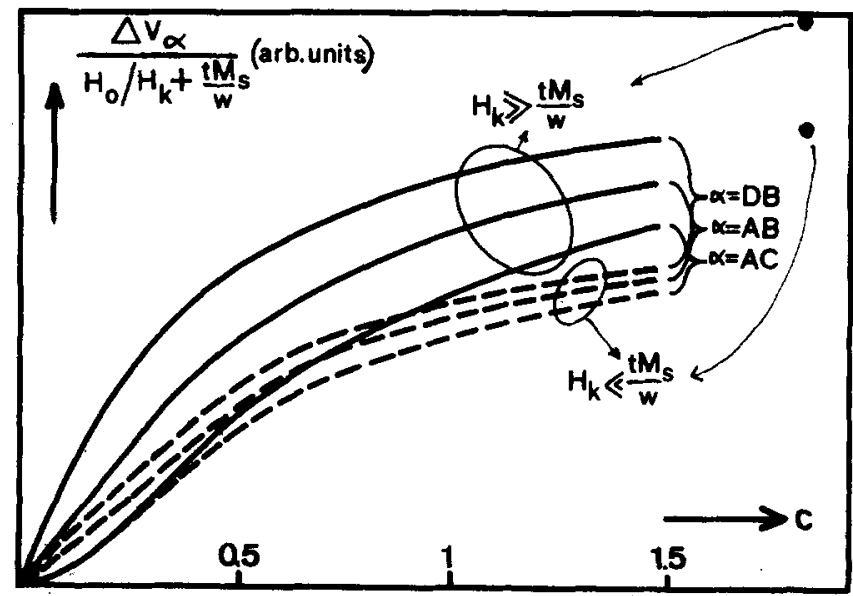

FIG.5. "One-sided" and traditional Hall sensitivities to exponential field $\mathrm{H}=\mathrm{H}_{0} \exp (-\mathrm{y} / \mathrm{cw})$ as a function of $\mathrm{c}$. The field amplitude is normalized to $\mathrm{H}_{\mathrm{k}}+\mathrm{tM} / \mathrm{w}$. Isolated points at right side indicate the situation that $c=\infty$. 
and for the limiting case that $\phi \rightarrow \pi / 2$ :

$$
\begin{aligned}
& \Delta \mathrm{H}_{\mathrm{y}}=\frac{1}{2} \mathrm{H}_{\mathrm{k}} \cos ^{2} \phi=\frac{1}{2} \mathrm{H}_{\mathrm{k}}\left(\mathrm{M}_{\mathrm{x}} / \mathrm{M}_{\mathrm{s}}\right)^{2} \\
& \Delta \mathrm{H}_{\mathrm{x}}=\frac{1}{2} \mathrm{H}_{\mathrm{k}} \cos ^{3} \phi=\frac{1}{2} \mathrm{H}_{\mathrm{k}}\left(\mathrm{M}_{\mathrm{x}} / \mathrm{M}_{\mathrm{s}}\right)^{3}
\end{aligned}
$$

$\Delta \mathrm{H}_{\mathrm{Y}}$ relates to a variation of $\mathrm{H}\left(=\mathrm{H}_{\mathrm{y}}\right)$ parallel to $\mathrm{H}$, while $\Delta \mathrm{H}_{\mathrm{x}}$ relates to the transverse case. With respect to the parallel susceptibility, magneto resistivity and $\mathrm{Hall}$ resistivity at $\mathrm{H}=\mathrm{H}_{\mathrm{k}}$ we have:

$$
\begin{aligned}
& \Delta M_{y} / \Delta H_{y}=-M_{s} / H_{k} \\
& \Delta \rho_{M^{\prime}} / \Delta H_{y}=-2\left(\rho_{H}-\rho_{\perp}\right) / H_{k} \\
& \Delta \rho_{H} / \Delta H_{y}=-\left(2 / H_{k}\right)^{1 / 2}\left(\Delta H_{y}\right)^{-1 / 2}
\end{aligned}
$$

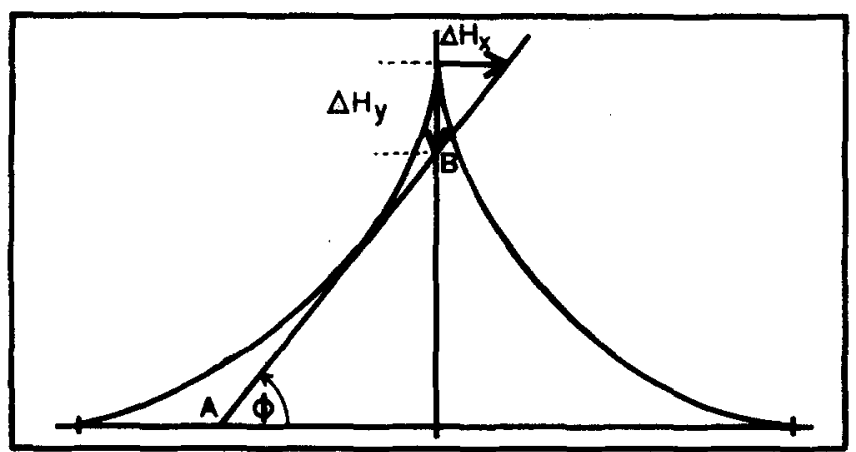

FIG.6. Geometrical aid to derive the formulas concerning the singularity at $\mathrm{H}_{\mathrm{r}}=\mathrm{H}_{\mathrm{k}}$. Note that use is made of the property that $|A B|=\mathrm{H}_{\mathrm{K}}$ for all tangents to the astroid.

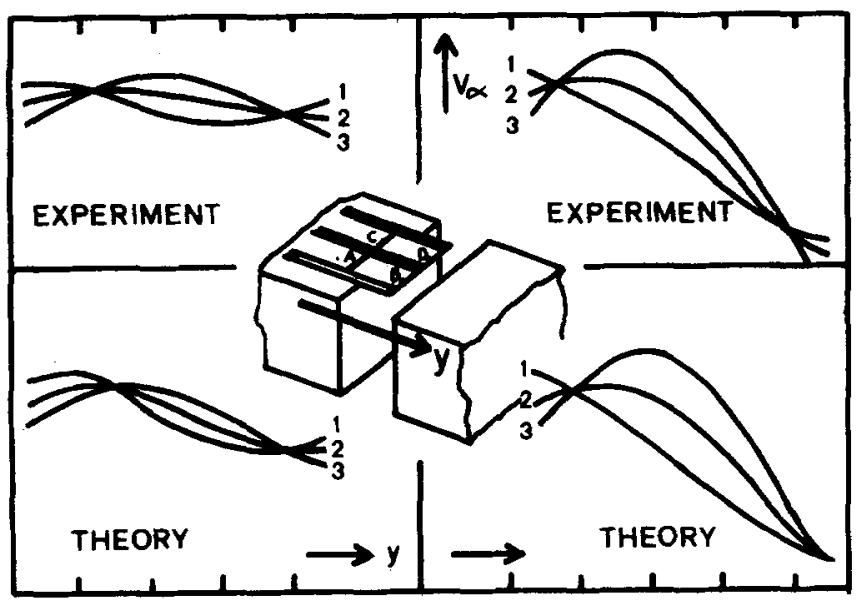

FIG.7. Comparison of theory and experiment (inset). Leftand right-hand curves are obtained for different values of the drive current of the magnet. Curves $1: V_{D B}$, curves $2: \mathrm{V}_{\mathrm{AB}}$ and curves $3: \mathrm{V}_{\mathrm{AC}}$.

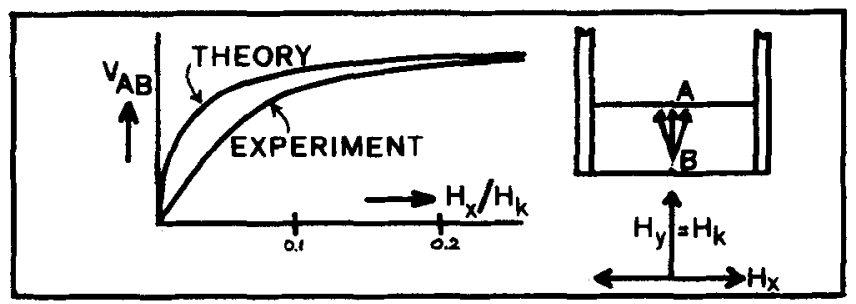

FIG.8. Response of the transducer to a longitudinal field $\mathrm{H}$ when biased at $\mathrm{H}=\mathrm{H}_{\mathrm{H}}$. The theoretical curve has an infinite slope at the orlgin.
In the transverse case we have:

$$
\begin{aligned}
& \Delta \mathrm{M}_{\mathrm{x}} / \Delta \mathrm{H}_{\mathrm{x}}=\mathrm{M}_{\mathrm{s}}\left(2 / \mathrm{H}_{\mathrm{k}}\right)^{1 / 3}\left(\Delta \mathrm{H}_{\mathrm{x}}\right)^{-2 / 3} \\
& \Delta \rho_{\mathrm{M}} / \Delta \mathrm{H}_{\mathrm{X}}=\left(\rho_{J}-\rho_{\perp}\right)\left(2 / \mathrm{H}_{\mathrm{K}}\right)^{2 / 3}\left(\Delta \mathrm{H}_{\mathrm{x}}\right)^{-1 / 3} \\
& \Delta \rho_{\mathrm{H}} / \Delta \mathrm{H}_{\mathrm{x}}=\left(\rho_{/}-\rho_{\perp}\right)\left(2 / \mathrm{H}_{\mathrm{k}}\right)^{1 / 3}\left(\Delta \mathrm{H}_{\mathrm{x}}\right)^{-2 / 3}
\end{aligned}
$$

It is seen that in the transverse case all effects are singular at $\mathrm{H}=\mathrm{H}_{\mathrm{k}}$, while in the longitudinal case only the Hall ${ }^{\mathrm{y}}$ resistivity shows a singularity.

\section{EXPERIMENTS}

We have performed some experiments to test the foregoing theories. In one experiment we used a permalioy sample with a thickness of $69 \mathrm{~nm}$. a width of $4 \mathrm{~mm}$. and a length of $5 \mathrm{~mm}$. (both parts). Since it was not very easy to produce exponentially decaying fields, we have performed the measurements in the fringe field of $a$ ring-type electro magnet with rectangular pole shoes (see inset in figure 7). The fringe fields have been measured beforehand with the help of a commercial Hall probe which is small with respect to the field gradients. With these field values and with the procedure described in the section on theory, we have computed the responses as a function of position in the $y$-direction. Compared with the response curves from the experiment there is a good agreement, at least qualitatively (fig.7). The differences which show up in a more quantitative comparison may be explained by the experimental error and/or the simplicity of our theoretical models. At the moment we consider the agreement as a sufficient proof of our starting points. With respect to the singularity in the transverse Hall resistivity we have performed a simple experiment, using the same specimen. With the help of a pair of Helmholtz coils an AC-field of small amplitude was generated in the direction of the strip axis. With the help of a second pair of Helmholtz coils a DC-field was generated perpendicular to the strip axis but in the plane of the strip. On increasing this DC-field a sharp, resonance like peak in the output could be observed. The amplitude of the peak output showed extreme sensitivity for misallignement, stray fields, etc., as can be expected. Fig. 8 gives a plot of the theoretical and experimental output amplitude as a function of the AC field amplitude, under the condition of "resonance". In spite of the expected inhomogeneities (e.g. dispersion) and the simple experimental procedure, a sensitivity could be obtained of $75 \mathrm{nV} / \mathrm{A} / \mathrm{m}$ ( $6 \mathrm{mV} / \mathrm{Oe})$, while under the same condition the planar Hall sensitivity at $H=0$ proved to be $9 \mathrm{nV} / \mathrm{A} / \mathrm{m}(0.7 \mathrm{mV} / \mathrm{Oe})$.

\section{CONCLUSION}

We have studied the planar Hall effect theoretically and have found the differences depending on the method of detection ("one-sided" and conventional) and the values of the several parameters. Our conclusion is that "one-sided" detection is applicable and of advantage in some practical situations. Note that "onesided" detection also presents a simple method to trim the currents to have $\mathrm{V}_{\mathrm{H}}=0$ at $\mathrm{H}=0$. Experiments have confirmed the theory. Also the expected singularity in the transverse $\mathrm{Hall}$ resistivity could easily be demonstrated. A sensitive device may be designed based on this effect.

\section{REFERENCES}

1.J.H.J.Fluitman, J.Phys.E. (Sci.Instr .) , 13, 783, 1980 2.F.G.West, J.AppI.Phys. $34,1171,1963$

3.J.H.J.Fluitman, IEEE Trans . Magn., MAG-14, 433, 1978 4.I.Hlasnik \& J.Kokavec,Sol.St.Electr. $9,585,1966$

5.H.Wieder, Hall generators and magnetoresistors, Pion Itd. ,London, 1971, Ch.3.

6.e.g:S.Middelhoek, Thesis Univ.Amsterdam, 1961. 